\title{
Metabolic Phenotyping of Blood Plasma by Proton Nuclear Magnetic Resonance to Discriminate between Colorectal Cancer, Breast Cancer and Lung Cancer
}

Robby Louis ${ }^{1}$, Evelyne Louis ${ }^{1}$, Kirsten Stinkens ${ }^{1,2}$, Liesbet Mesotten ${ }^{1,3}$, Eric de Jonge ${ }^{4}$, Michiel Thomeer ${ }^{1,2}$, Philip Caenepeel ${ }^{1,5}$ and Peter Adriaensens ${ }^{6 *}$

${ }^{1}$ Faculty of Medicine and Life Sciences, Hasselt University, 3590 Diepenbeek, Belgium ${ }^{2}$ Department of Respiratory Medicine, Ziekenhuis Oost-Limburg, 3600 Genk, Belgium ${ }^{3}$ Department of Nuclear Medicine, Ziekenhuis Oost-Limburg, 3600 Genk, Belgium ${ }^{4}$ Department of Gynaecology, Ziekenhuis Oost-Limburg, 3600 Genk, Belgium

${ }^{5}$ Department of Gastroenterology, Ziekenhuis Oost-Limburg, 3600 Genk, Belgium

${ }^{6}$ Applied and Analytical Chemistry, Institute for Materials Research, Hasselt University, 3590 Diepenbeek, Belgium

\begin{abstract}
Background: Although many studies have demonstrated that plasma metabolic phenotyping allows discriminating between cancer patients and controls, it remains unclear whether different cancer types elicit distinguishable metabolic signatures. Therefore, the present study was designed to examine whether metabolic phenotyping of blood plasma by proton nuclear magnetic resonance spectroscopy allows to discriminate between 37 colorectal cancer, 37 breast cancer and 37 lung cancer patients.

Material and methods: Plasma proton nuclear magnetic resonance spectra were rationally divided into 110 integration regions defined on the basis of spiking experiments with known metabolites. The normalized integration values of these 110 regions, which represent the metabolic phenotype, were used as statistical variables to construct a classification model which enables to discriminate between the three aforementioned cancer types.

Results: The resulting model allows to classify $78 \%$ of the colorectal cancer patients, $95 \%$ of the breast cancer patients and $84 \%$ of the lung cancer patients correctly.

Conclusion: This preliminary feasibility study provides strong indications that the plasma metabolic phenotype has potential to become a complementary diagnostic tool to differentiate between cancer types in addition to known general cancer biomarkers.
\end{abstract}

Keywords: Colorectal cancer; Breast cancer; Lung cancer; Metabolic phenotype; Blood plasma; ${ }^{1} \mathrm{H}-\mathrm{NMR}$ spectroscopy

Abbreviations: ${ }^{1} \mathrm{H}$ : Proton; BMI: Body Mass Index; NMR: Nuclear Magnetic Resonance; OPLS-DA: Orthogonal Partial Least Squares Discriminant Analysis; PCA: Principal Component Analysis; PET: Positron Emission Tomography; ZOL: Ziekenhuis Oost-Limburg

\section{Introduction}

It is widely accepted that cancer cells exhibit a major reprogramming of their energy metabolism in order to fulfill the high metabolic demands that are associated with increased cell proliferation and survival [1]. Because the metabolic alterations in cancer cells provoke changes in the metabolic phenotype of the patient, metabolites might serve as attractive biomarkers for facilitating the diagnosis of cancer. Complex mixtures of metabolites in biofluids, such as plasma, serum or urine, can be mined for diagnostic biomarkers by means of the metabolomics approach. This discipline represents a relatively new 'omics' science downstream of genomics, transcriptomics and proteomics that uses an analytical platform in conjunction with multivariate pattern recognition approaches in order to discover and monitor metabolic changes in patient biospecimens related to disease status or in response to a medical or external intervention [2]. Since metabolites are the end products of all cellular regulatory processes, their levels can be regarded as the ultimate response of biological systems to genetic, biological and/or environmental perturbations [3]. More specifically, alterations in the concentrations of metabolites are the net result of epigenetic changes, genetic variation and changes in the activity and/or levels of enzymes [4]. The metabolome, i.e. the complete set of metabolites present in the human body, is therefore the most closely related to the observed phenotype and provides the most accurate representation of the functional status of the patient when compared with the genome, transcriptome and proteome [5]. Consequently, metabolomics holds great promise for early cancer detection as the concentrations of metabolites are sensitive to subtle changes in the pathological status of the patient, such as the early onset of tumor growth [6].

One of the main analytical tools that is commonly used in metabolomics to identify and quantify a wide range of metabolites in biological samples is proton nuclear magnetic resonance ( $\left.{ }^{1} \mathrm{H}-\mathrm{NMR}\right)$ spectroscopy. This technique only requires minimal sample preparation, needs no chemical derivatization, can be easily automated and is fast, non-destructive, highly reproducible and relatively cheap on a per sample basis, making it a promising platform for performing high-throughput diagnostic analyses on a large scale [5,7]. In the past decade, a growing interest in the diagnostic utility of ${ }^{1} \mathrm{H}$-NMR-based

*Corresponding author: Adriaensens P, Applied and Analytical Chemistry Institute for Materials Research, Hasselt University, 3590 Diepenbeek, Belgium Tel: +3211268396; E-mail: peter.adriaensens@uhasselt.be

Received September 09, 2016; Accepted September 27, 2016; Published September 30, 2016

Citation: Louis R, Louis E, Stinkens K, Mesotten L, de Jonge E, et al. (2016) Metabolic Phenotyping of Blood Plasma by Proton Nuclear Magnetic Resonance to Discriminate between Colorectal Cancer, Breast Cancer and Lung Cancer. Metabolomics (Los Angel) 6: 187. doi: 10.4172/2153-0769.1000187

Copyright: $\odot 2016$ Louis R, et al. This is an open-access article distributed under the terms of the Creative Commons Attribution License, which permits unrestricted use, distribution, and reproduction in any medium, provided the original author and source are credited. 
Citation: Louis R, Louis E, Stinkens K, Mesotten L, de Jonge E, et al. (2016) Metabolic Phenotyping of Blood Plasma by Proton Nuclear Magnetic Resonance to Discriminate between Colorectal Cancer, Breast Cancer and Lung Cancer. Metabolomics (Los Angel) 6: 187. doi: 10.4172/2153-0769.1000187

Page 2 of 5

metabolomics of blood plasma has emerged in the field of oncology, and various studies have already established the potential of this methodology to detect various cancer types [8,9]. However, since the majority of these studies only compared the plasma metabolic phenotype of cancer patients with that of controls, it remains unclear whether different tumors share the same metabolic perturbations or whether certain metabolic alterations are specific for certain cancer types. Recently, we have demonstrated that metabolic phenotyping of plasma by ${ }^{1} \mathrm{H}-\mathrm{NMR}$ spectroscopy allows to discriminate between breast cancer and lung cancer, classifying $99 \%$ of 80 female breast cancer patients and $93 \%$ of 54 female lung cancer patients correctly. These results were successfully validated in an independent cohort in which $82 \%$ of 60 female breast cancer patients and $89 \%$ of 81 male lung cancer patients were correctly classified [10]. In order to further explore the ability of the plasma metabolic phenotype to differentiate between cancer types, the present study aims to investigate whether ${ }^{1} \mathrm{H}$-NMR-based metabolomics of plasma allows to discriminate between colorectal cancer, breast cancer and lung cancer.

\section{Material and Methods}

\section{Subjects}

Thirty-seven colorectal cancer patients and an equal number of breast cancer and lung cancer patients, all with an adenocarcinoma, were randomly selected in order to construct a classification model which permits to discriminate between the different cancer types based on the metabolic phenotype of blood plasma. The colorectal cancer patients were included in the Limburg Positron Emission Tomography (PET) Center (Hasselt, Belgium), at the Gastroenterology Department of Ziekenhuis Oost-Limburg (ZOL, Campus Sint-Jan, Genk, Belgium) and in the Center for Specialized Medicine (Reumacentrum, Genk, Belgium). The diagnosis of colorectal adenocarcinoma was confirmed by histopathological examination of biopsies taken during endoscopy. The lung cancer patients were recruited in the Limburg PET center and the diagnosis of lung adenocarcinoma was confirmed by means of a pathological biopsy or by a medical doctor specialized in the interpretation of radiological and clinical data regarding lung cancer. The stage of both the colorectal and lung tumors was defined according to the $7^{\text {th }}$ edition of the tumor, nodes and metastases classification of malignant tumors. The breast cancer patients were included at the day of primary surgery at the Gynaecology Department of ZOL. The diagnosis of breast adenocarcinoma was confirmed by a core needle biopsy and the stage of the tumors was defined by the revised staging system for breast cancer [11]. For each group of cancer patients, blood samples were collected prior to surgery, chemotherapy or any other cancer treatment. In addition, blood collection and sample preparation were performed according to a fixed protocol and by trained staff. Furthermore, for each group, the following exclusion criteria were defined: 1) Poorly controlled diabetes (fasting blood glucose $\geq 200$ $\mathrm{mg} / \mathrm{dL}$ ); 2) Any inflammatory condition; 3) Any other cancer type besides colorectal cancer, breast cancer or lung cancer; 4) History or treatment of cancer during the past five years; 5) Medication intake on the morning of blood sampling; and 6) No fasting for at least six hours prior to blood sampling. All procedures were conducted in accordance with the ethical rules of the Helsinki declaration and Good Clinical Practice and the study protocols were approved by the Medical Ethics Committees of ZOL and Hasselt University (Campus Diepenbeek, Hasselt, Belgium). All participants provided written informed consent prior to their inclusion. The studies in which the patients were enrolled are registered at clinicaltrials.gov (trial registration numbers: NCT02364154 and NCT02362776).

\section{Sample collection, sample preparation and ${ }^{1} \mathrm{H}-\mathrm{NMR}$ analysis}

Detailed protocols regarding blood sample collection, sample preparation and ${ }^{1} \mathrm{H}-\mathrm{NMR}$ analysis have been previously described elsewhere [10].

\section{Statistical analysis}

Multivariate statistics were performed using SIMCA- $\mathrm{P}^{+}$(version 14.0, Umetrics, Umea, Sweden). After the ${ }^{1} \mathrm{H}-\mathrm{NMR}$-derived variables had been subjected to mean centering and Pareto scaling, Orthogonal Partial Least Squares Discriminant Analysis (OPLS-DA) was performed to construct a classification model which discriminates between the colorectal cancer, breast cancer and lung cancer patients based on their metabolic phenotype [12]. The validity of the established model was evaluated based on 1) the total amount of variation between and within the three groups explained by the model (denoted as $\mathrm{R}^{2} \mathrm{Y}$ (cum) and $\mathrm{R}^{2} \mathrm{X}$ (cum), respectively) and 2) the predictive ability of the model as determined by sevenfold cross-validation (denoted as $\mathrm{Q}^{2}$ (cum)). Furthermore, permutation testing was performed to ensure that the discrimination between the three cancer types was not due to overfitting of the data. Additionally, Principal Component Analysis (PCA) was carried out to identify possible confounding effects of age, gender, Body Mass Index (BMI) or tumor stage on group discrimination.

\section{Results and Discussion}

\section{Subject characteristics}

The demographical and clinical characteristics of the colorectal cancer, breast cancer and lung cancer patients are displayed in Table 1. All breast cancer patients were female, while the majority of colorectal cancer $(70 \%)$ and lung cancer patients (57\%) were male. However, PCA revealed that there was no apparent clustering with respect to gender in the entire patient cohort, indicating that the discrepancy in gender among the three different groups of cancer patients is not a confounding factor (Figure 1A). Additionally, there were no clusters observed when PCA score plots of the entire patient cohort were stained according to age, BMI or tumor stage, thereby confirming that none of these factors have a confounding effect on the discrimination between colorectal cancer, breast cancer and lung cancer patients (Figures 1B-1D).

\section{Discrimination between colorectal cancer, breast cancer and lung cancer based on the plasma metabolic phenotype}

The OPLS-DA model that was established to discriminate between the three groups of cancer patients allows to classify 29 out of 37 colorectal cancer patients $(78 \%), 35$ out of 37 breast cancer patients (95\%) and 31 out of 37 lung cancer patients (84\%) correctly ( $\mathrm{R}^{2} \mathrm{X}$ (cum): 0.875 and $\mathrm{R}^{2} \mathrm{Y}$ (cum): 0.561) (Figure 2A). The predictive ability of the model is relatively high $\left(\mathrm{Q}^{2}(\mathrm{cum})=0.387\right)$, indicating that the discrimination between the three cancer types based on the metabolic phenotype is valid. The validity of the OPLS-DA model was also confirmed by permutation testing, which demonstrated that the $\mathrm{R}^{2}(\mathrm{cum})$ and $\mathrm{Q}^{2}$ (cum) values of the actual model are higher than those of twenty permuted models (Figure 2B). Although the number of subjects included in this study is still limited, these results strongly suggest that colorectal cancer, breast cancer and lung cancer patients display different metabolic alterations in blood plasma, independent of tumor stage.

In conclusion, the present study demonstrates that metabolic phenotyping of plasma by ${ }^{1} \mathrm{H}-\mathrm{NMR}$ spectroscopy allows to discriminate between the three most common cancer types worldwide, i.e., lung 
Citation: Louis R, Louis E, Stinkens K, Mesotten L, de Jonge E, et al. (2016) Metabolic Phenotyping of Blood Plasma by Proton Nuclear Magnetic Resonance to Discriminate between Colorectal Cancer, Breast Cancer and Lung Cancer. Metabolomics (Los Angel) 6: 187. doi: 10.4172/2153-0769.1000187

Page 3 of 5

\begin{tabular}{|c|c|c|c|}
\hline & Colorectal cancer patients & Breast cancer patients & Lung cancer patients \\
\hline \multicolumn{4}{|c|}{ Demographical characteristics } \\
\hline Number of subjects, $n$ & 37 & 37 & 37 \\
\hline \multicolumn{4}{|l|}{ Gender } \\
\hline Male, n (\%) & $26(70)$ & $0(0)$ & $21(57)$ \\
\hline Female, $\mathrm{n}(\%)$ & $11(30)$ & $37(100)$ & $16(43)$ \\
\hline Age (years, range) & $65 \pm 10(45-86)$ & $57 \pm 10(42-78)$ & $65 \pm 10(43-88)$ \\
\hline \multicolumn{4}{|l|}{ Smoking status } \\
\hline Active smoker, n (\%) & $4(11)$ & $8(22)$ & $18(49)$ \\
\hline Quit smoking >6 months, $\mathrm{n}(\%)$ & $20(54)$ & $0(0)$ & $16(43)$ \\
\hline Never smoked, n (\%) & $10(27)$ & $0(0)$ & $3(8)$ \\
\hline Unknown, n (\%) & $3(8)$ & $29(78)$ & $0(0)$ \\
\hline \multicolumn{4}{|c|}{ Clinical characteristics } \\
\hline $\mathrm{BMI}\left(\mathrm{kg} / \mathrm{m}^{2}\right.$, range $)$ & $\begin{array}{c}26.9 \pm 4.5 \\
(21.4-40.8)\end{array}$ & $\begin{array}{c}25.7 \pm 3.1 \\
(20.2-35.4)\end{array}$ & $\begin{array}{c}25.6 \pm 4.8 \\
(17.0-35.8)\end{array}$ \\
\hline \multicolumn{4}{|l|}{ Tumor laterality } \\
\hline Right, n (\%) & - & $16(43)$ & $23(62)$ \\
\hline Left, n (\%) & - & $19(51)$ & $12(32)$ \\
\hline Bilateral, n (\%) & - & $2(6)$ & $0(0)$ \\
\hline Unknown, n (\%) & - & $0(0)$ & $2(6)$ \\
\hline \multicolumn{4}{|l|}{ Tumor location $^{a}$} \\
\hline Cecum, n (\%) & $4(10)$ & - & - \\
\hline Ascending colon, n (\%) & $1(2)$ & - & - \\
\hline Hepatic flexure, n (\%) & $2(5)$ & - & - \\
\hline Descending colon, n (\%) & $1(2)$ & - & - \\
\hline Sigmoid colon, n (\%) & $9(23)$ & - & - \\
\hline Rectosigmoid junction, n (\%) & $7(18)$ & - & - \\
\hline Rectum, n (\%) & $16(40)$ & - & - \\
\hline \multicolumn{4}{|l|}{ Tumor stage $\mathrm{e}^{\mathrm{a}, \mathrm{b}}$} \\
\hline $0, \mathrm{n}(\%)$ & $1(2)$ & $0(0)$ & $0(0)$ \\
\hline $\mathrm{I}, \mathrm{n}(\%)$ & $10(25)$ & $22(56)$ & $8(21)$ \\
\hline II, n (\%) & $8(20)$ & $16(41)$ & $4(11)$ \\
\hline III, n (\%) & $13(33)$ & $1(3)$ & $11(30)$ \\
\hline IV, n (\%) & $6(15)$ & $0(0)$ & $14(38)$ \\
\hline Unknown, n (\%) & $2(5)$ & $0(0)$ & $0(0)$ \\
\hline \multicolumn{4}{|l|}{ Tumor histopathology $y^{\mathrm{a}, \mathrm{b}}$} \\
\hline Adenocarcinoma, n (\%) & $40(100)$ & $39(100)$ & $37(100)$ \\
\hline \multicolumn{4}{|l|}{ Tumor receptor status $^{b}$} \\
\hline \multicolumn{4}{|l|}{ Estrogen receptor } \\
\hline Positive, n (\%) & - & $33(85)$ & - \\
\hline Negative, n (\%) & - & $6(15)$ & - \\
\hline \multicolumn{4}{|l|}{ Progesterone receptor } \\
\hline Positive, n (\%) & - & $30(77)$ & - \\
\hline Negative, n (\%) & - & $9(23)$ & - \\
\hline \multicolumn{4}{|l|}{ HER2 } \\
\hline Positive, n (\%) & - & $3(8)$ & - \\
\hline Negative, n (\%) & - & $36(92)$ & - \\
\hline
\end{tabular}

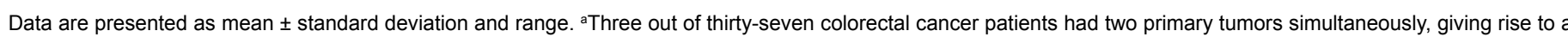
total number of forty colorectal tumors. ${ }^{\text {T}}$ Two out of thirty-seven breast cancer patients had two primary tumors simultaneously, rendering the total number of breast tumors at thirty-nine. HER2: Human Epidermal Growth Factor Receptor 2

Table 1: Demographical and clinical characteristics of the colorectal cancer, breast cancer and lung cancer patients. 
Citation: Louis R, Louis E, Stinkens K, Mesotten L, de Jonge E, et al. (2016) Metabolic Phenotyping of Blood Plasma by Proton Nuclear Magnetic Resonance to Discriminate between Colorectal Cancer, Breast Cancer and Lung Cancer. Metabolomics (Los Angel) 6: 187. doi: 10.4172/2153-0769.1000187

A

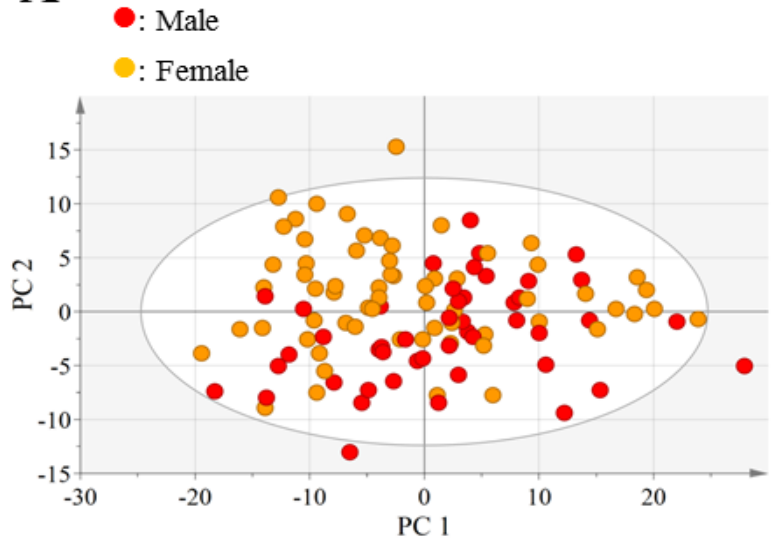

C

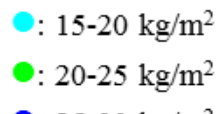

: $25-30 \mathrm{~kg} / \mathrm{m}^{2}$

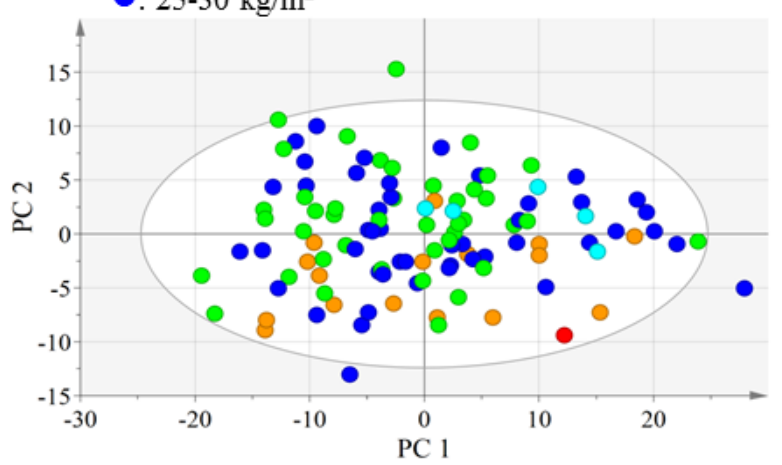

B
: $40-50$ years
: $70-80$ years
: $50-60$ years
•: $80-90$ years

: $60-70$ years

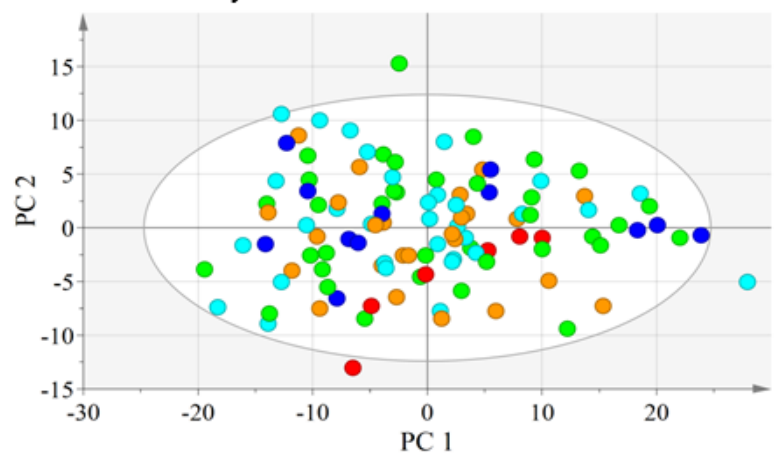

D

: stage 0

: stage III

: stage I

: stage IV

-: stage II

: stage unknown

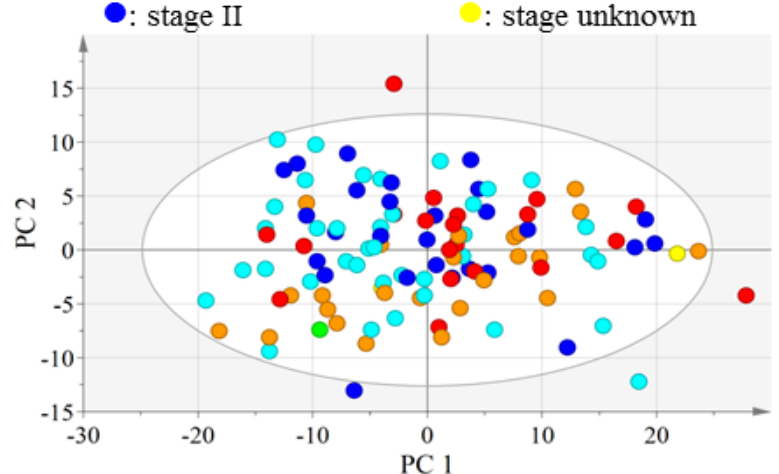

Figure 1: Search for possible confounding factors in the discrimination between colorectal cancer, breast cancer and lung cancer based on the metabolic phenotype of blood plasma determined by ${ }^{1} \mathrm{H}-\mathrm{NMR}$ spectroscopy. PCA score plots of the entire patient cohort stained according to A) gender, B) age, C) BMI, and D) tumor stage; PC: Principal Component.

A

- Colorectal cancer patients

: Breast cancer patients

$\Delta$ : Lung cancer patients

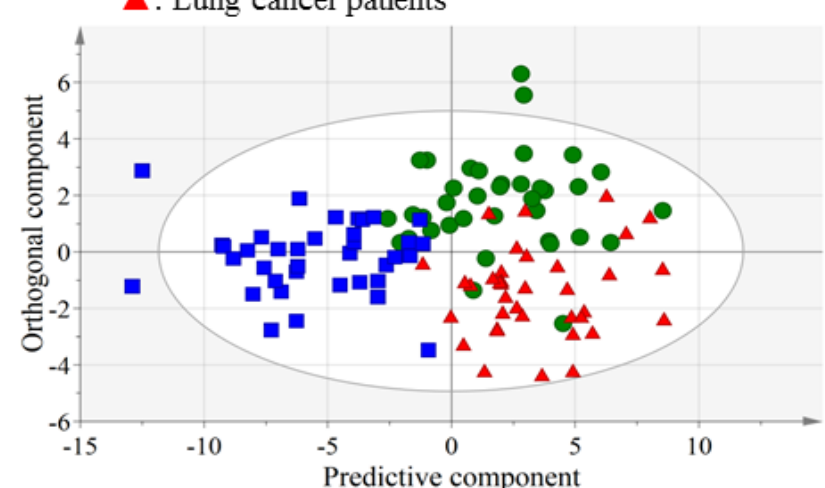

B

: $\mathrm{R}^{2}(\mathrm{cum})$

口: $\mathrm{Q}^{2}(\mathrm{cum})$

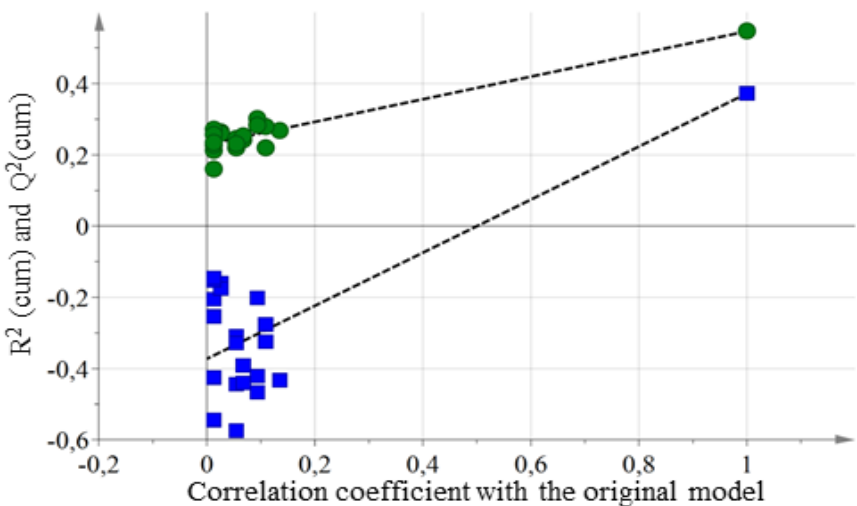

Figure 2: Discrimination between colorectal cancer, breast cancer and lung cancer based on the ${ }^{1} \mathrm{H}-\mathrm{NMR}$-derived metabolic phenotype of blood plasma. A) OPLS-DA score plot discriminating between 37 colorectal cancer patients and an equal number of breast cancer and lung cancer patients, all with an adenocarcinoma, based on their metabolic phenotype. The model has two predictive components, which describe the variation between the three groups, and five orthogonal components, which display the variation within the different groups. B) Permutation plot comparing the goodness of fit of the constructed OPLS-DA model with that of twenty permuted models. The vertical axis displays the $\mathrm{R}^{2}$ (cum) and $\mathrm{Q}^{2}$ (cum) values of the actual model and the permuted models, while the horizontal axis shows how well each model is correlated with the actual model. The $R^{2}(\mathrm{cum})$ and $\mathrm{Q}^{2}(\mathrm{cum})$ values of the actual model are located at the right, whereas those of the permuted models are located at the left. $R^{2}$ (cum) denotes the variation explained by the model, while $\mathrm{Q}^{2}$ (cum) is the predictive ability of the model as determined by sevenfold cross-validation. 
Citation: Louis R, Louis E, Stinkens K, Mesotten L, de Jonge E, et al. (2016) Metabolic Phenotyping of Blood Plasma by Proton Nuclear Magnetic Resonance to Discriminate between Colorectal Cancer, Breast Cancer and Lung Cancer. Metabolomics (Los Angel) 6: 187. doi: 10.4172/2153-0769.1000187

Page 5 of 5

cancer, breast cancer and colorectal cancer, respectively. Although it has already been demonstrated for several types of malignancies that cancer patients can be differentiated from controls on the basis of their plasma metabolic phenotype, the present study indicates that, in addition, different cancer types display distinct metabolic characteristics rather than a general cancer metabolome. Keeping in mind that the sample size in this study is still somewhat limited and an independent validation study should be performed, the promising results of this study offer additional incentives to further investigate the diagnostic utility of ${ }^{1} \mathrm{H}$-NMR-based metabolomics of plasma for different cancer types.

\section{Acknowledgements}

This study is part of the 'Limburg Clinical Research Program (LCRP) UHasselt-ZOL-Jessa', supported by the foundation Limburg Sterk Merk, province of Limburg, and Flemish government, Hasselt University, ZOL and Jessa Hospital. We thank the Research Foundation Flanders for their support via the MULTIMAR project. Furthermore, we would like to thank Dr. K. Vanhove, Dr. K. Vandeurzen, Dr. K. Darquennes and Dr. P. Jorissen for their help in the recruitment of subjects for this study. In addition, we would also like to thank G. Reekmans for his assistance in the ${ }^{1} \mathrm{H}-\mathrm{NMR}$ analysis of plasma samples.

\section{References}

1. Hanahan D, Weinberg RA (2011) Hallmarks of cancer: the next generation. Cell 144: 646-674.

2. Beger RD (2013) A review of applications of metabolomics in cancer Metabolites 3: 552-574.
3. Ma Y, Zhang $P$, Yang $Y$, Wang F, Qin H (2012) Metabolomics in the fields of oncology: a review of recent research. Mol Biol Rep 39: 7505-7511.

4. Griffin JL, Shockcor JP (2004) Metabolic profiles of cancer cells. Nat Rev Cancer 4: 551-561.

5. O'Connell TM (2012) Recent advances in metabolomics in oncology Bioanalysis 4: 431-451.

6. Gu H, Gowda GA, Raftery D (2012) Metabolic profiling: are we en route to better diagnostic tests for cancer? Future Oncol 8: 1207-1210.

7. Mamas M, Dunn WB, Neyses L, Gooadcre R (2011) The role of metabolites and metabolomics in clinically applicable biomarkers of disease. Arch Toxico 85: 5-17.

8. Duarte IF, Gil AM (2012) Metabolic signatures of cancer unveiled by NMR spectroscopy of human biofluids. Prog Nucl Magn Reson Spectrosc 62: 51-74.

9. Louis E, Bervoets L, Reekmans G, de Jonge E, Mesotten L, et al. (2015) Phenotyping human blood plasma by $1 \mathrm{H}-\mathrm{NMR}$ : a robust protocol based on metabolite spiking and its evaluation in breast cancer. Metabolomics 11:225-236.

10. Louis E, Adriaensens P, Guedens W, Vanhove K, Vandeurzen K, et al. (2015) Metabolic phenotyping of human blood plasma: a powerful tool to discriminate between cancer types? Ann Oncol 27: 178-184.

11. Singletary SE, Allred C, Ashley P, Bassett LW, Berry D, et al. (2002) Revision of the American Joint Committee on Cancer staging system for breast cancer. J Clin Oncol 20: 3628-3636.

12. Trygg J, Wold S (2002) Orthogonal projections to latent structures (O-PLS). J Chemometr 16: 119-128. 